Rev. salud pública. 14 sup (1): 112-121, 2012

\title{
Aborto voluntario y actividad laboral. Reflexiones para el debate
}

\author{
Induced abortion and labor activity. Reflections for discussion
}

María E. Orjuela-Ramírez

Departamento de Salud de Colectivos. Facultad de Enfermería, Universidad Nacional de Colombia. Bogotá. meorjuelar@unal.edu.co

Recibido 8 Mayo 2010/Enviado para Modificación 5 Noviembre 2010/Aceptado 4 Abril 2011

\section{RESUMEN}

El aborto voluntario es un fenómeno global que según distintos autores responde a patrones de comportamientos construidos socialmente y donde influyen las realidades sociales particulares de cada país. Este fenómeno requiere la información necesaria para comprender el complejo proceso que conduce a la decisión de la mujer para optar por la interrupción del embarazo y saber entender las circunstancias sociales, económicas y sanitarias que pueden explicar esta determinación. Con este propósito se presenta para el debate, algunas consideraciones sobre el aborto voluntario y la actividad laboral de las mujeres que optan por esta práctica, con especial mención la situación en España. Los argumentos están sustentados en el análisis de las estadísticas de interrupción Voluntaria del embarazo (IVE) reportadas por el Ministerio de Sanidad y Política Social, la participación de la mujer en el mercado laboral en España obtenidas del Instituto Nacional de Estadística (INE), los resultados de investigación sobre la asociación entre la situación laboral de la mujer y la Interrupción Voluntaria del embarazo y la revisión exhaustiva de las literatura científica sobre las distintas perspectivas del abordaje del aborto voluntario. Merece especial importancia el análisis de la actividad laboral de la mujer, como un posible determinante en la decisión de la mujer para interrumpir su embarazo, variable que ha sido identificada en la mayoría de las investigaciones como una de las condiciones socioeconómicas en las mujeres que optan por esa alternativa, al considerar que el embarazo interfiere con el empleo de las mujeres o, bien, les impide emplearse.

Palabras Clave: Aborto inducido, trabajo de mujeres (fuente: DeCS, BIREME).

\section{ABSTRACT}

The induced abortion is a global phenomenon that according to various authors respond to socially constructed patterns of behavior and where they influence social realities of each country. This phenomenon requires the information necessary to understand the complex process leading to the decision of women to opt for abortion and able to understand the social, economic and health that can explain this requirement. 
For this purpose is presented for discussion, some considerations on voluntary abortion and labor activity of women who opt for this practice, with special mention of the situation in Spain. The arguments are supported by statistical analysis of the voluntary interruption of pregnancy (IVE) reported by the Ministry of Health and Social Policy, participation of women in the labor market in Spain obtained from the National Statistics Institute (INE), the research results on the association between employment status of women and voluntary termination of pregnancy and comprehensive review of scientific literature on the different perspectives of the approach of voluntary abortion. Analysis deserves special importance of women's work activity as a possible factor in the decision of women to terminate their pregnancies, a variable that has been identified in most of the investigations as a socioeconomic condition for women who choose for that alternative, considering that pregnancy interferes with the employment of women or, rather, prevents them from use.

Key Words: Induced abortion, employment status (fuente: Mesh, NLM).

$\mathrm{E}$ 1 aborto voluntario se define como la terminación deliberada del desarrollo del feto durante el embarazo, antes de que haya alcanzado las 20 semanas de gestación. En este caso, también denominado interrupción voluntaria del embarazo, puede ser clandestino o legal, según el marco jurídico vigente en cada país. El aborto inducido puede ser terapéutico o médico, cuando se realiza para evitar riesgos en la vida o la salud de la mujer. Según el diccionario de la Real Academia Española (RAE), abortar es la interrupción del embarazo por causas naturales o deliberadamente provocadas. Desde la ética científica, aborto es la interrupción de la vida del ser humano, en forma natural o provocada, en cualquiera de las etapas de cigoto, embrión o feto $(1,2)$.

En España, las estadísticas del Ministerio de Sanidad y Política Social indican que de las 41910 interrupciones voluntarias del embarazo realizadas en 1991, se ha pasado a 91664 en el 2005. La tasa de interrupción voluntaria del embarazo en el año 2005 se situó en 9,60 por cada 1000 mujeres en edad fértil (15-44 años), frente al 4,79 del año 1991. Es decir, que en los últimos 15 años se ha duplicado la tasa de incidencia ${ }^{1}$. Este hecho se presenta también en el conjunto de los países de la Unión Europea, en los que la tasa de interrupciones voluntarias del embarazo por mil nacidos vivos ha pasado de 199,8 en 1991 a 227,6 en el año 2003. En los últimos 10 años (1993 al 2003) se han incrementado de 781837 a 850212 interrupciones del embarazo, lo que representa un aumento aproximado del $9 \%$ (3). 
Una de las razones de ese incremento puede ser la tendencia creciente en los últimos años de participación de la mujer en el mercado laboral en la mayoría de los países de la Unión Europea y, sobre todo, en España. Dado que las mujeres que se deciden por la IVE señalan como uno de los motivos de esa práctica el hecho de evitar dificultades en el empleo, ese aumento podría estar relacionado con el incremento en la tasas de actividad laboral

Es evidente, la tendencia creciente de la participación de la mujer en el mercado laboral en la mayoría de los países de la Unión Europea y, sobre todo, en España, donde el porcentaje de participación de la mujer entre los 15 y 49 años de edad han pasando de 46,6 \% en 1991 al 58,4 \% en 2005 (4). Este incremento podría estar relacionado con el aumento en las tasas de la interrupción voluntaria del embarazo observadas, ya que esa evolución muestra una tendencia casi paralela con la tasa de participación laboral durante el mismo período.

Otro hecho de especial relevancia es la importante aportación de la inmigración femenina al mercado de trabajo en España, que de acuerdo con algunos investigadores se debe a la llegada de población inmigrante. De hecho, la tasa de ocupación femenina, en el caso de las mujeres extranjeras, ha pasado del 53,6 \% en 2001 al 60,5 \% en 2005. Dado que algunos estudios sugieren que la frecuencia de la interrupción voluntaria del embarazo en la población inmigrante es mucho más alta que en la población autóctona española, una parte del incremento en los índices del aborto voluntario en la última década podría ser atribuido al contexto social, laboral y demográfico que la inmigración ha conformado en España (5).

El aborto voluntario es una práctica que ha estado presente de diferentes maneras y con distintas características en la historia de las sociedades humanas. Es por lo tanto un fenómeno global, si bien los motivos para abortar no son universales ni comunes a todas las mujeres. Así por ejemplo, el aborto voluntario se ha utilizado como práctica anticonceptiva para espaciar, limitar o posponer los embarazos no planeados. Los diversos motivos pueden variar según la calidad de los servicios de información, educación, programas de planificación familiar. Dependen en muchos casos del significado de la maternidad, así como de la aceptación social de embarazos fuera de las uniones legales socialmente establecidas. Esta situación puede ser agudizada en sectores y grupos de población de adolescentes y jóvenes, residentes en áreas rurales e indígenas, por las mayores barreras de índole muy variada que enfrentan. 
Distintos motivos, apuntan a las carencias materiales, sociales, culturales y afectivas del entorno social y familiar de la mujer para asumir la crianza de los hijos y que la llevan a recurrir al aborto voluntario. La escasez de los medios económicos suficientes para asegurar el cuidado de la familia o no poseer el apoyo familiar o del Estado para el cuidado de los hijos, sobre todo para quienes tienen un trabajo de tiempo completo o doble jornada, son circunstancias frecuentes para las adolescentes y para mujeres que no cuentan con autonomía financiera o recursos propios. También, las relaciones no estables o de conflicto con la pareja, la presión de los padres, la estigmatización y rechazo social y moral por un embarazo fuera del matrimonio, son causas importantes para recurrir al aborto.

Algunas razones tienen que ver directamente con el desarrollo personal y las propias expectativas y proyectos de vida de las mujeres, como la imposibilidad de seguir los estudios a causa del embarazo, por lo cual, se puede optar por el aborto para continuar su educación o consolidar una carrera laboral. Otros factores que repercuten en una mayor incidencia del aborto, se asocian con el mejoramiento del nivel educativo de las mujeres y su creciente participación en el mercado de trabajo.

Son muy pocos los estudios, que han analizado el fenómeno. En España, se puede destacar, el estudio realizado por la demógrafa Margarita Delgado, investigadora del Consejo Superior de Investigaciones Científicas (CSIC), en el que analiza los determinantes de la interrupción voluntaria del embarazo (IVE, entre los años 1991 y 2001, en mujeres en edad fértil (15 a 49 años) residentes en España (6). Determina en su estudio, el perfil de las mujeres que recurren al aborto a partir de diferentes variables como la situación laboral, número de hijos, estado civil, nivel de instrucción, tamaño del lugar de residencia. Entre los principales determinantes de la interrupción voluntaria del embarazo, la autora destaca sobre todo la situación laboral. Las mujeres que trabajan fuera del hogar, y en particular las que lo hacen por cuenta ajena, son las que muestran una incidencia más alta del aborto voluntario. La cifra absoluta de mujeres trabajadoras que optaron por el aborto inducido, casi se duplicó en el decenio estudiado. El factor laboral está además íntimamente relacionado con el número de hijos. Se establece que a mayor actividad laboral, tiende a ser menor el número de hijos, en parte, según muestra el estudio, por ser mayor entre las mujeres que trabajan fuera del hogar las que en más alto porcentaje recurren al IVE. 
Destacan las autoras que se observa una mayor tendencia a la opción del Aborto voluntario en mujeres con menor número de hijos, con mayor nivel de instrucción, lo que se vincula con los cambios sociales acontecidos en el transcurso de los últimos años.

De otra parte, un informe del Ministerio de Sanidad y Política Social, señala que en el 2005, aumentó el porcentaje de asalariadas y disminuyó el de mujeres sin empleo que recurren al aborto voluntario. Persiste el aumento del aborto voluntario entre mujeres con menor nivel educativo, apreciándose disminuciones significativas entre quienes tienen mayor nivel de estudios (7).

La inmigración es una situación íntimamente ligada con el aborto voluntario, ya que la información disponible de estudios sociológicos realizados en mujeres, ha demostrado que factores socioeconómicos tales como nivel de estudios, el idioma, el empleo, el estado civil, el contexto cultural, la educación sexual y reproductiva, el acceso a servicios de salud y métodos de planificación familiar, contribuyen a determinar el número de embarazos y abortos indeseados en una sociedad $(8,9)$.

De acuerdo con el estudio "Análisis del impacto económico de la inmigración femenina latinoamericana en la economía española” (10), realizado por el Instituto de la mujer, el fuerte incremento de la oferta de trabajo extranjera se corresponde con la alta demanda que presenta el mercado laboral español. La población inmigrante en edad de trabajar que ha venido a engrosar la población potencialmente activa del mercado de trabajo, ha experimentado un incremento firme y sostenido desde mediados de la década pasada, cuando se ha pasado de un total de 400000 personas en el año 1996, hasta alcanzar la cifra de 3324000 en el año 2005. De los más de 4,3 millones de empleos creados en España durante el periodo 19992005, el 38,8 \% han sido ocupados por población extranjera proveniente de países en desarrollo o subdesarrollados.

El efecto más relevante derivado del claro predominio de población extranjera en edad laboral es, sin lugar a dudas, el incremento notable de población activa. Resulta evidente, que los inmigrantes que eligen España como destino lo hacen en su amplia mayoría, por razones de índole económica y, en ese sentido, tienen como objetivo prioritario su incorporación formal o informal al mercado de trabajo nacional. Los 
sectores donde predominantemente se concentra la población inmigrante femenina, es el sector servicios (el 89,5 \%). El detalle de ocupaciones muestra que la mayoría de las mujeres inmigrantes se encuentran empleadas como trabajadoras no cualificadas en el sector servicios (46,5 \%) y también como trabajadoras de los servicios de restauración (26,8 \%) o dependientes de comercio (5,6 \%). Cabe destacar que a pesar de que la población extranjera ocupa principalmente empleos de baja cualificación, el nivel de educación que presentan las mujeres extranjeras, no es precario, aunque sí inferior al de las mujeres españolas. Así, al analizar el nivel de formación que presenta la población ocupada femenina, un 79,9 \% ha concluido por lo menos algún nivel de secundaria y un $17 \%$ tiene estudios universitarios de grado medio o superior.

La ocupación de inmigrantes en las tareas de servicio doméstico, cuidado de niños y ancianos en España, es un fenómeno que se explica, por una mayor demanda de este tipo de trabajadoras en el marco de la evolución económica y social de la población española. Este tipo de tareas, que en otros países desarrollados son asumidas por un mejor reparto de tareas en las familias y gracias a la ayuda del Estado de Bienestar, están realizándose en el país, gracias al empleo de mano de obra de bajo costo. Merece la pena destacar, que aproximadamente un tercio de los hogares donde se contrata inmigrantes como personal domestico, el ama de casa, se puede incorporar al mercado laboral, es decir, cambia su situación laboral de inactividad a actividad laboral.

En nuestras sociedades, el empleo es la principal vía de acceso a los recursos monetarios para alcanzar unos estándares de vida dignos y por lo tanto asegurar la independencia económica, que a su vez, representa para las mujeres un cauce de participación social. El aumento de la proporción de mujeres en la fuerza de trabajo y la reducción de la brecha en las tasas de participación femenina y masculina ha sido una de las tendencias más relevantes detectadas en los mercados laborales en los últimos años. El incremento de participación de la mujer en la fuerza de trabajo ha comportado además, importantes transformaciones en los roles y responsabilidades de hombres y mujeres en la esfera familiar y el trabajo remunerado, en un contexto donde la actividad definidora de lo femenino, asigna a la mujer las tareas domésticas privadas y al varón las tareas productivas públicas. El trabajo se considera una relación social que varía de significado dependiendo del contexto y características del mismo. 
Así por ejemplo, cuando este se desarrolla en el interior de un grupo social primario, como la familia, se trata de tareas incluidas en el ámbito privado, que está supeditado a las relaciones interpersonales que se Desde este punto de vista, la participación femenina en el mercado laboral, ha significado para la mujer una forma importante de participar en la vida pública a través de instituciones u organizaciones de más amplio carácter. Lo cual ha sido un factor clave de integración social y connotada de prestigio (posición social) en las posibilidades de desarrollar la autoestima, la autorrealización individual y condicionar relaciones de confianza y sociabilidad para la mujer. En un sentido más amplio, representa una actividad productiva remunerada y generadora de derechos propios frente a los derivados que le produce el rol de ama de casa. Además de proporcionar la ampliación de elecciones de estilos de vida e incluso la construcción de un sentido de la propia existencia.

Desde otro punto de vista, las decisiones de participación laboral femenina son motivadas, en parte, por las características de ocupaciones que se caracterizan por una menor necesidad de uso de la fuerza física, con horarios y jornadas más flexibles, condiciones factibles de incorporación femenina al mundo del trabajo. Aunque también constituye una expresión de la proliferación de estrategias generadoras de ingreso, mediante las cuales las mujeres contribuyen a sostener el nivel de vida de sus familias. El aumento en las tasas de participación económica de las mujeres ha significado el incremento en el mercado laboral de mujeres en edad adulta y la pérdida de la relación entre estado civil y participación laboral. De igual manera, el crecimiento del empleo terciario, se relaciona con el incremento observado en la participación laboral de las mujeres en la medida que ha ampliado oportunidades laborales y especialmente porque ha facilitado la compatibilización de la vida familiar y profesional (11).

Apesar de las transformaciones productivas y tecnológicas, de la igualdad jurídica entre hombres y mujeres, las políticas de ordenamiento laboral, la mujer suele estar representadas en el empleo flexible, probablemente porque se sigue considerando que su responsabilidad primera sigue siendo ocuparse del bienestar de la familia.

En su gran mayoría las mujeres que se encuentran insertas en el mercado laboral continúan siendo las responsables del trabajo que se realiza en los hogares, el que conforma la denominada “economía del cuidado”, expresión 
que alude al conjunto de bienes y servicios no remunerados que prestan las mujeres de todas las edades al interior de las familias. Se les asocia menos con contratos de trabajo estándar a tiempo completo a partir del cual se han construido la consideración social del mercado de trabajo (12).

De acuerdo con los resultados un estudio reciente, “Asociación entre la situación laboral de la mujer. España 1991-2005” (13) se ha descrito que las tasas de aborto voluntario según situación laboral de la mujer, evidencian el crecimiento de estas cifras tanto en mujeres activas como en inactivas. Concretamente, la tasa por mil mujeres, en activas ha crecido de 5,73 en 1991 a 9,39 en el año 2005, en comparación las tasas en inactivas eran 3,08 en 1991 y 5,72 en 2005. Los autores del estudio, refieren que el aumento en la tasa de abortos voluntarios, puede estar relacionada con la presencia cada vez mayor de la población femenina en el mercado de trabajo y el incremento notable de mujeres jóvenes (20-29 años) en la población en edad de trabajar. Ello ha generado un crecimiento de la población activa entre las mujeres sin estudios universitarios, como resultado del proceso de desarrollo económico y social que ha venido experimentando España en las últimas décadas.

En consecuencia, diversas publicaciones señalan 50-51 que la decisión de participación de la mujer en el mercado laboral y las determinaciones concernientes a su fecundidad y, más concretamente, a la interrupción del embarazo en mujeres activas o inactivas están estrechamente ligadas. Sin embargo, las decisiones de la mujer en cuanto al cuidado de los hijos y la participación en el mercado laboral pueden resultar hasta cierto punto incompatibles, porque las dos actividades compiten por su tiempo de dedicación. Además, implica decidir no sólo cuántos hijos tener a lo largo de la vida biológica fértil, sino el momento del ciclo vital cuando deba tenerlos. En este sentido, en la medida que transcurre el tiempo en cada etapa de su vida y se modifica el conjunto de información que la mujer condiciona a sus decisiones, esta puede ir modificando sus determinaciones futuras de participar en el mercado laboral o de tener el número de hijos deseados.

Estudios referenciados en la literatura científica $(14,15)$ sugieren que el creciente aumento de las tasas de actividad laboral femenina no ha implicado una reorganización sustantiva de las tareas domésticas dirigida a lograr un reparto más equilibrado de éstas entre hombres y mujeres. 
Así, un alto número de mujeres siguen siendo quienes se ocupan de las tareas domésticas y responsabilidades familiares, bien como actividad exclusiva, bien como prolongación de la jornada laboral. Por consiguiente, la conciliación de la vida laboral y familiar genera un conflicto, por tener que hacer frente a las obligaciones que comporta tener una familia, sin la pérdida de oportunidades en la vida laboral o el menoscabo del rol profesional. Pero dado la coincidencia del tiempo en momentos decisivos para la formación de la familia y la consolidación de la situación laboral, es posible que las mujeres estén optando por permanecer en el mercado de trabajo, antes que dedicarse a la crianza de los hijos.

Los dilemas que rodean al aborto inducido requieren la búsqueda de mayor evidencia científica para comprender los factores determinantes que inducen a la mujer para optar por este procedimiento y saber entender la influencia de las circunstancias sociales, económicas y sanitarias que pueden explicar las razones que inducen a las mujeres a optar por el aborto Voluntario.

En este contexto, la conciliación de la vida laboral y familiar genera un conflicto en la mujer por la coincidencia de las oportunidades determinantes para la formación de la familia y la crianza de los hijos y paralelamente para la consolidación de la carrera profesional. En estas circunstancias se puede optar por abandonar el mercado de trabajo para dedicarse a la familia o priorizar la mejora y consolidación de la situación profesional o laboral. Por lo anterior, es decisivo el papel del Estado en la regulación de este conflicto entre el trabajo y la familia. Buscar la protección de la maternidad implica el doble objetivo de proteger la salud y garantizar los derechos que se derivan del empleo. Es necesario que la contratación en función de una maternidad potencial o actual no suponga una práctica discriminatoria que interfieran en la decisión de interrumpir el embarazo o

\section{REFERENCIAS}

1. Valenzuela CY. Ética científica del aborto terapéutico. Rev méd Chile. 2003; 5:562-568.

2. Diccionario de la lengua española. $22^{\mathrm{a}}$ ed. Editorial Espasa Calpe; 2001. Madrid.

3. Ministerio de Sanidad y Consumo. Dirección General de Salud Pública. Interrupción Voluntaria del Embarazo, Datos definitivos correspondientes a 2005. 2006; 1:17-20.

4. Larraña M, Echevarria C. Actividad laboral femenina en España e igualdad de oportunidades entre mujeres y hombres. Revista del Ministerio de Trabajo y Asuntos Sociales. 2004; 55: 65-81. 
5. Ministerio de Trabajo y Asuntos Sociales [Internet]. Boletín Estadístico de Extranjería e Inmigración [actualizado 26 May 2007;]. Disponible en: http://extranjeros.mtin.es/ es/InformacionEstadistica/Boletines/Archivos/BoletindeExtranjeria-num-13-Web. pdf Consultado: 24 sep 2007

6. Delgado M. La evolución reciente de la fecundidad y el embarazo en España: la influencia del aborto. Revista Española de Investigaciones Sociológicas.1999;(87) 83-116.

7. Lete I, Martínez M. La salud reproductiva: datos y reflexiones. Gac sanit.2004; 18(Supl 1):170-4.

8. Henshaw SK. Unintended pregnancy in United States. Fam Plann Perspect. 1998; 30(1): 24-9.

9. Bankole A, Singh S, Haas T. Characteristics of women who obtain induced abortion: A worldwide review. Int Fam Plan Perspect. 1999; 25:68-77

10. Ministerio de Trabajo y Asuntos Sociales. Análisis del impacto económico de la inmigración femenina latinoamericana en la economía española; 2005. p. 5-85

11. Miller LM. Participación laboral femenina y Estados de bienestar. Documentos de trabajo (Instituto de Estudios Sociales Avanzados de Andalucía).2004; 3:2-27.

12. Rico MN. El trabajo de las mujeres. Amenazas, seguridades y necesidad de políticas públicas. Notas para un debate. Seminario CIEPP-DSE - Precariedad laboral, vulnerabilidad social y seguridad socioeconómica. Buenos Aires: Comisión Económica para América Latina y el Caribe, 2001.

13. Orjuela ME, Ronda E, Regidor E. Asociación entre situación laboral de la mujer y la interrupción voluntaria del embarazo en España, 1991-2001. Gac Sanit. 2007; 21(Supl 2):5-65.

14. Gálvez N. La conciliación de la vida laboral y familiar como proyecto de calidad de vida desde la igualdad. RES.2005; 5: 91-107.

15. Maroto G, García M, Rodríguez I. El reto de la maternidad en España: dificultades sociales y sanitarias. Gac Sanit. 2004; 18 (Supl 2)13-26. 